\title{
Novel Amphiphilic Carriers: One Step Further in Cancer Management
}

\author{
*SumairaNaeem ${ }^{1,2}$, LikVoonKiew ${ }^{3}$, Lip Yong Chung ${ }^{4}$, GeethaViswanathan ${ }^{4}$ and ${ }^{*}$ Misni Bin Misran ${ }^{1}$ \\ ${ }^{1}$ Department of Chemistry, University of Malaya, Malaysia \\ ${ }^{2}$ Department of Chemistry, University of Gujarat, Pakistan \\ ${ }^{3}$ Department of Pharmacology, University of Malaya, Malaysia \\ ${ }^{4}$ Department of Pharmacy, University of Malaya, Malaysia
}

Submission: May 20, 2016; Published: June 23, 2016

*Corresponding author: Sumaira Naeem, Misni Bin Misran, Department of Chemistry, Faculty of Science, University of Malaya, 50603, Kuala Lumpar, Malaysia, Email: misni@um.edu.my; sumairamaan@gmail.com

\begin{abstract}
The ideal goal of treatment, and most often the goal in practice, is the successful removal of cancer with near-zero damage to the rest of the body. As cancer is referred to a class of disease, it is unlikely to idealize a single cure for cancer. The attempt to cure cancer has been revolutionized over decades as the underlying processes have revealed more horizons of understanding. Although a number of strategies have been developed, the associated side effects have led the research to enter a new era of nanoengineering and nanotechnology. Designing versatile nanocarriers offer efficient drug delivery applications with enhanced therapeutic benefits of drug. Amphiphilic nanocarriers fall under the heading of such novel carriers which offer high circulation half-life, low risk to benefit ratio of drugs, sustained release and effective targeting. They provide a paradigm for the design of nanocarriers with a broad spectrum of functionality, applicability, and versatility of synthetic drug delivery particles.
\end{abstract}

Keywords: Nanotechnology; Pharmaceutical Carriers; Targeted Delivery; Intracellular Drug Delivery; Liposomes

\section{Mini Review}

Out of different choices of therapy like surgery, chemotherapy, radiation therapy, hormonal therapy, or targeted therapy; the selection criteria is specified with reference to the tumor location \& grade, stage of disease and above all the general health condition of the patient. Therefore, a number of experimental strategies are under development for complete cure of cancer with as minimum side effects of the anticancer drugs as possible. For instance, present-day cancer therapy involves application of catheters for chemotherapy to destroy cancerous cells. Catheters, thin tubes of medical grade materials, are inserted into the body to treat diseases and performing surgical procedures. The method follows the initial chemotherapy which renders shrinkage of any cancer present to; surgical removal of the tumor if possible, followed by more chemotherapies and radiations over the last 25 years but still needs more improvement due to rapid cancer cell growth. Current research offers new dosing routes via blood vessels to fuel tumor growth with more specific targeting actions. Whatever the route or treatment may be, the eventual expectation is the improved change in patient's quality of life and least damage to the normal healthy tissues.
Unfortunately, the treatments covering all oncological specifications are still not good enough to kill the cancer cells before the cancer kills the patient. The advancement in nanotechnology had bridged the gap by offering different drug delivery carriers with a hope to move us away from near-toxic doses of non-specific agents [1,2].

Non-specific distribution of cytotoxic drugs may lead to variety of toxic effects by killing both normal and malignant cells [3]. Controlled release, understanding target structure and function, achievement of the maximum therapeutic effect, minimized risk to benefit ratios have been the major factors in designing novel drug delivery systems in the past few decades [4]. Nanotechnology has been utilized to treat a variety of disease and disorders and its applications can be traced in physics, chemistry, communication, robotics, biology and medicine $[5,6]$.

Oral and parental administration routes have been considered as traditional drug administration routes. The former is a type whereby the administered drug gets absorbed from the gut followed by its admittance in blood stream, whereas the later route is a method of direct drug injection into the blood possible only in solution or emulsion form. Once injected as solution, it gets distributed into the body according to its partition 
coefficient and become easily accessible to degrading factors like water and enzymes. Such problems have been controlled by entrapping such drugs into some potential particulate carrier system.

Colloidal systems, being at the epicenter of modern drug delivery systems, have long been explored for their applications in cancer management. A variety of colloidal nanoparticles have been proposed as carriers like composite nano-shells, dendrimers, viral nanoparticles, magnetics nanoparticles, quantum dots, micelles and liposomes [7]. Among these, drug encapsulation into liposomes resulted in increased circulation times, increased drug deposition to tumor tissues by decreasing drug metabolic degradation, and their altered tissue distribution in the body.

Liposomes come under the heading of amphiphilic carriers. The term amphiphile describes a chemical compound possessing both hydrophilic (polar) and lipophilic (non-polar) properties. Such a compound is named as amphiphilic or amphipathic $[8,9]$. The amphiphilic compound tends o partition when placed in an immiscible biphasic system containing both aqueous and organic solvents. The extent of partitioning is evaluated by the extent of hydrophobic and hydrophilic portion of that compound. Liposomes prepared by phospholipids have gained tremendous attention as novel and efficient drug delivery carriers. Phospholipids are very important amphiphiles and are found as main components of the biological membranes where they define the way of membrane formation [10]. They position as bilayers whereby their polar groups are exposed towards the surrounding aqueous medium and lipophilic chains are sandwiched between polar head groups throughout the bilayer arrangement. Some other important amphiphiles present in the biological membranes are cholesterol, required to maintain both membrane structural integrity and fluidity [11] and glycolipids, to serve as markers for cellular recognition and to provide energy [12]. Many other amphiphilic compounds, for instance pepducins $[13,14]$ act as intracellular modulators of signals from receptors to $G$ proteins which are involved in transferring signals from a no of stimuli outside a cell to its interior [15].

Therefore, being self-assembled amphiphiles, liposome can create the possibility to dissolve and protect anticancer drugs from adverse external effects, and then make sure to access cancer targeted sites by leaving blood under specified triggered release strategies [16]. Some of the exemplary carriers have also been introduced to clinical practice and are FDA approved like Doxil and Abraxane. Clinical trials have opened new era for the patients to choose the best option which may increase survival time and the quality of life by killing cancer before the cancer kills the patients [17].

Drug delivery required designing liposomes in such a way so as to optimize carriers with reference to drug loading, controlled drug release rate, rapid clearance by reticuloendothelial system, and above all the drug delivery across cell membranes to intracellular sites of action [18]. The nanoparticles are purposely engineered ranging from a few $\mathrm{nm}$ to several hundred $\mathrm{nm}$ so as to pass through the fenestration of the leaky cancer endothelium. All the Nano-vehicles are constructed on the basis of their use and preferential accumulation at tumor sites with fewer accumulations in liver, lungs, spleen, and bone marrow [19]. It is very important to take a look at tumor environment for better understanding of it cure and problem solving aspects. The notion of Tumor vasculature, and Tumor interstitium, Cellular resistance is important to understand abnormal cell growth phenomenon $[20,21]$ resistance to the inward flux of molecules \& enhancedpermeability and retention (EPR) effect [22-24], and to understand cellular resistance mechanisms $[25,26]$.

Liposomes have gained special attention with reference to their many clinical applications like gene delivery [27] diagnostic imaging, vaccines, some pharmaceutical uses of stealth liposomes as vesicular containers, in particular for Liposome-encapsulated hemoglobin (LEH) developed as oxygen therapeutic. The developed PEG-LEH had a circulation half-life of about 65 hours.

Carrier targeting to specific organs or different tumor types is a greatest challenge. Nanoparticles should selecticley and specifically transport drug payloads to cancerous tissues like breast cancer [28,29] receptors for liver targeting [30] and folate receptors [31] and the therapeutic agents will thereby be delivered to the interior of the cancer cells.

Undoubtedly, innovation in the field of synthetic drug delivery biomaterials has gained fundamental importance in terms of functionality and diversity, it still cannot reach the complexity and sophistication levels exhibited by distinctive biological moieties. Erythrocytes, as remarkable biological entities, have been investigated many a times for understanding their complex biological functionality when it comes to mimic their unique characteristics [32-34]. Three different strategies have been followed to mimic erythrocytes i.e. Red Blood Cell (RBC) membrane ghosts [35] Engineered RBC as synthetic carriers [36] and RBC-membrane clocked vehicles [37]. However, drug delivery is a growing interdisciplinary field which covers new materials and their interaction with the selected drugs, innovation in their biomedical properties, and improved therapeutic mode of actions with much reduced toxic effects.

\section{Acknowledgment}

The authors would like to acknowledge and express their sincere gratitude to the Ministry of Education, Malaysia, for the High Impact Research (Grant No. UM-MOHE:UM.C/625/1/HIR/ MOHE//SC/09), HIR-MOHE grants (UM.C/625/1/HIR/MOHE/ MED/17 and UM.C/625/1/HIR/MOHE/MED/33), University of Malaya Research (grant no. FL001F-13 BIO), Post Graduate Research Fund (Grant No.PG094-2014A) and Post Graduate Research Fund (Grant No.PG123- 2012Bg). The authors would also like to acknowledge University of Gujarat, Gujarat, Pakistan. 
The authors pay their gratitude to the University of Malaya Bright Sparks Unit for financially supporting this work.

\section{References}

1. Brannon Peppas L, Blanchette JO (2004) Nanoparticle and targeted systems for cancer therapy. Adv Drug Deliv Rev 56(11): 1649-1659.

2. Bagalkot V, Deiuliis JA, Rajagopalan S, Maiseyeu A (2016) "Eat me" imaging and therapy. Adv Drug Deliv Rev 99(Pt A): 2-11.

3. Ishida T, Kiwada H, (2015) Agent for enhancing anti-tumor effect comprising oxaliplatin liposome preparation, and anti-tumor agent comprising the liposome preparation. Google Patents.

4. Naeem S, Kiew LV, Yong CL, Yin YT, Bin Misran M (2015) Drug delivery and innovative pharmaceutical development in mimicking the red blood cell membrane. Reviews in Chemical Engineering 31 (5): 491508.

5. Esposito S, Colicchia S, de la Torre X, Donati F, Mazzarino M, et al. (2016) Liposomes as potential masking agents in sport doping. Part 2: Detection of liposome-entrapped haemoglobin by flow cytofluorimetry. Drug Test Anal.

6. Psarros C, Lee R, Antonopoulos A, Margaritis M, Antoniades C (2016) Nanomedicine in Cardiovascular. Nanotechnology and Drug Delivery, Volume Two: Nano-Engineering Strategies and Nanomedicines against Severe Diseases: 257.

7. Bigdeli A, Palchetti S, Pozzi D, Hormozi Nezhad MR, Baldelli Bombelli F, et al. (2016) Exploring Cellular Interactions of Liposomes Using Protein Corona Fingerprints and Physicochemical Properties. ACS Nano 10(3): 3723-3737.

8. Xiao D, Jia HZ, Ma N, Zhuo RX, Zhang XZ (2015) A redox-responsive mesoporous silica nanoparticle capped with amphiphilic peptides by self-assembly for cancer targeting drug delivery. Nanoscale 7(22): 10071-10077.

9. Suzuki R, Omata D, Oda Y, Unga J, Negishi Y, et al. (2016) Cancer Therapy with Nanotechnology-Based Drug Delivery Systems: Applications and Challenges of Liposome Technologies for Advanced Cancer Therapy. Nanomat Pharmaco: 457-482.

10. Li Y, Lin J, Yang X, Li Y, Wu S, et al. (2015) Self-Assembled Nanoparticles Based on Amphiphilic Anticancer Drug-Phospholipid Complex for Targeted Drug Delivery and Intracellular Dual-Controlled Release. ACS Appl Mater Interfaces 7(32): 17573-17581.

11. Thakur N, Abhilash C, Vivek K, Nitan B, Neeraj B (2016) Liposome: Emerging Trends In Transdermal Drug Delivery. World Journal of Pharmacy and Pharmaceutical Sciences 5(5): 511-525.

12. Goyard D, Shiao T C, Fraleigh N, Vu H-Y, Lee H, et al. (2016) Expedient synthesis of functional single-component glycoliposomes using thiolyne chemistry. J Mater Chem B S1-S52.

13. Di L (2015) Strategic approaches to optimizing peptide ADME properties. AAPS J (1): 134-143.

14. Carr R, Benovic JL (2016) From biased signalling to polypharmacology: unlocking unique intracellular signalling using pepducins. Biochem Soc Trans 44(2): 555-561.

15. Tsvetanova NG, Irannejad R, von Zastrow M (2015) G proteincoupled receptor (GPCR) signaling via heterotrimeric $G$ proteins from endosomes. J Biol Chem 290(11): 6689-6696.

16. Koudelka S, Mikulik R, Masek J, Raska M, Turanek Knotigova P, et al. (2016) Liposomal nanocarriers for plasminogen activators. J Control Release 227: 45-57.

17. Halwani M, Hossain Z, Khiyami MA, Omri A (2015) Liposomal [Beta]Glucan: Preparation, Characterization and Anticancer Activities. J Nanomed \& Nanotech 6(5): 1.
18. Allen TM, Cullis PR (2013) Liposomal drug delivery systems: from concept to clinical applications. Adv Drug Deliv Rev 65(1): 36-48.

19. Haley B, Frenkel E (2008) Nanoparticles for drug delivery in cancer treatment. Urol Oncol 26(1): 57-64.

20. Eberhard A, Kahlert S, Goede V, Hemmerlein B, Plate KH, et al. (2000) Heterogeneity of angiogenesis and blood vessel maturation in human tumors: implications for antiangiogenic tumor therapies. Cancer Res 60(5): 1388-1393.

21. Baban DF, Seymour LW (1998) Control of tumour vascular permeability. Adv Drug Deliv Rev 34(1): 109-119.

22. Jain RK (2001) Delivery of molecular and cellular medicine to solid tumors. Adv Drug Deliver Rev 46(1-3): 149-168.

23. Jang SH, Wientjes MG, Lu D, Au JL (2003) Drug delivery and transport to solid tumors. Pharm Res 20(9): 1337-1350.

24. Maeda H (2010) Tumor-selective delivery of macromolecular drugs via the EPR effect: background and future prospects. Bioconjug Chem 21(5): 797-802.

25. Links M, Brown R (1999) Clinical relevance of the molecular mechanisms of resistance to anti-cancer drugs. Expert Rev Mol Med 1999: 1-21.

26. Vasir JK, Labhasetwar V (2005) Targeted drug delivery in cancer therapy. Technol Cancer Res Treat 4(4): 363-374.

27. Mahato RI (2005) Water insoluble and soluble lipids for gene delivery. Adv Drug Deliv Rev 57(5): 699-712.

28. Becerril B, Poul MA, Marks JD (1999) Toward selection of internalizing antibodies from phage libraries. Biochem Biophys Res Commun 255(2): 386-393.

29. Nielsen UB, Kirpotin DB, Pickering EM, Hong K, Park JW, et al. (2002) Therapeutic efficacy of anti-ErbB2 immunoliposomes targeted by a phage antibody selected for cellular endocytosis. Biochim Biophys Acta 1591(1-3): 109-118.

30. Kim IS, Kim SH (2003) Development of polymeric nanoparticulate drug delivery systems: evaluation of nanoparticles based on biotinylated poly (ethylene glycol) with sugar moiety. Int J Pharm 245(1-2): 67-73.

31. Lu Y, Low PS (2003) Immunotherapy of folate receptor-expressing tumors: review of recent advances and future prospects. J Control Release 91(1-2): 17-29.

32. Gupta V, Doshi N, Mitragotri S (2013) Permeation of insulin, calcitonin and exenatide across Caco-2 monolayers: measurement using a rapid, 3-day system. PLoS One 8(2): e57136.

33. Roggers RA, Joglekar M, Valenstein JS, Trewyn BG (2014) Mimicking red blood cell lipid membrane to enhance the hemocompatibility of large-pore mesoporous silica. ACS Appl Mater Interfaces 6(3): 16751681.

34. Hosseini H, Li Y, Kanellakis P, Tay C, Cao A, et al. (2015) Phosphatidylserine liposomes mimic apoptotic cells to attenuate atherosclerosis by expanding polyreactive IgM producing B1a lymphocytes. Cardiovasc Res 106(3): 443-52.

35. Han Y, Wang X, Dai H, Li S (2012) Nanosize and surface charge effects of hydroxyapatite nanoparticles on red blood cell suspensions. ACS Appl Mater Interfaces 4(9): 4616-4622.

36. Nishit Doshia, Alisar S Zahra, Srijanani Bhaskarb, Joerg Lahannb, Samir Mitragotria (2009) Red blood cell-mimicking synthetic biomaterial particles. Proc Natl Acad Sci U S A 106(51): 21495-21499.

37. Fang RH, Hu CM, Chen KN, Luk BT, Carpenter CW, et al. (2013) Lipid-insertion enables targeting functionalization of erythrocyte membrane-cloaked nanoparticles. Nanoscale 5(19): 8884-8888. 